\title{
Improving respiratory outcomes after pediatric cardiac surgery: new uses for nitric oxide.
}

\author{
Jacques Scharoun ${ }^{1}$
}

${ }^{1}$ Weill Cornell Medicine

November 18, 2021

\begin{abstract}
Nitric oxide has been used to treat respiratory failure in post-pediatric cardiac surgical patients. High frequency oscillator ventilation is used to rescue infants who have failed conventional ventilation. In this issue a study is presented showing the benefit of combining these two therapies for improved outcomes in infants with hypoxic respiratory failure after congenital heart surgery

Title:
\end{abstract}

Improving respiratory outcomes after pediatric cardiac surgery: new uses for nitric oxide Author:

Jacques H Scharoun

Assistant Professor of Clinical Anesthesiology

Dept of Anesthesiology

Weill Cornell Medicine

New York Presbyterian Hospital

New York, NY, USA

Email: jhs2001@med.cornell.edu

Co-authors : None

Funding received : none

Data availability : No original data was used in creating this manuscript

Conflicts of Interest Statement : The author has no conflicts of interest to disclose

IRB approval : not applicable as there were no experimental subjects

Patient Consent : not applicable

ORCID ID : 0000-0002-7166-6593

Corresponding author: Please address all correspondence to:

Jacques H Scharoun

Assistant Professor of Clinical Anesthesiology 
Dept of Anesthesiology

Weill Cornell Medicine

New York Presbyterian Hospital

Email: jhs2001@med.cornell.edu

\section{Improving respiratory outcomes after pediatric cardiac surgery: new uses for nitric oxide.}

Congenital heart surgery is performed on tens of thousands of neonates and infants every year. Most of them are extubated within 3 days of surgery. However, $20 \%$ of these children remain intubated after 72 hours and $10 \%$ are still intubated after 7 days. ${ }^{1,2}$ Prolonged intubation can lead to ventilator acquired pneumonia, barotrauma, subglottic stenosis, and increased cost. Furthermore, while the mean intensive care unit (ICU) length of stay (LOS) for a neonate after cardiac surgery is 9 days, and 6 days for an infant, ${ }^{3} 9 \%$ will stay in the ICU for greater than 14 days. ${ }^{3}$ These outliers can have a disproportionate impact on hospital finances. Pasquali in 2014 found that 2 additional days in the ICU after ventricular septal defect (VSD) repair was associated with an increased cost of $\$ 10,661$, and those staying 1 week beyond the median incurred an extra $\$ 100,000$ in costs. ${ }^{4}$ Effective treatment or prevention of prolonged mechanical ventilation in post cardiac surgical infants is beneficial for both the patient and society. The study presented by Huang et $\mathrm{al}^{5}$ in this issue shows the advantage of combining 2 complementary treatments in post-cardiac surgical patients with respiratory failure.

Pagowska-Klimek in 2010 showed a tripling of mean ICU LOS in pediatric cardiac surgical patients with pulmonary hypertension (PHTN). ${ }^{3}$ PHTN also increases the risk of prolonged post-operative mechanical ventilation (>7 days) 3-fold. ${ }^{1}$ Infants with left to right shunts, such as VSD and atrioventricular canal, have increased pulmonary blood flow (PBF) and often elevated pulmonary arterial pressure, which predisposes them to develop PHTN. ${ }^{6}$ When these patients undergo cardiopulmonary bypass (CPB), afterwards they can have increased pulmonary vascular reactivity ${ }^{7}$ which leads to repeated episodes of acute elevation in pulmonary vascular resistance (PVR). This increase in right ventricle afterload worsens any ongoing right heart failure, leading to significant morbidity and delays weaning from the ventilator. ${ }^{6}$ Causes for this reactivity are theorized to include endothelial dysfunction from systemic inflammation triggered by the CPB machine as well as ischemic lung reperfusion injury. ${ }^{8}$ Even in the absence of pre-existing pulmonary hypertension, those with single ventricle physiology and passive PBF are particularly sensitive to changes in PVR and may benefit from pulmonary vasodilation ${ }^{8}$.

Optimizing pulmonary blood flow after congenital heart surgery is desirable, particularly in the subset of patients who have hypoxic respiratory failure requiring prolonged ventilation. Milrinone is commonly used for this purpose, but with 2 important caveats. First, it also causes systemic vasodilation, potentially leading to hypotension. ${ }^{8}$ Second, as an intravenous agent, its efficacy depends on its ability to reach the pulmonary vessels. In the setting of certain right to left shunts a portion of the milrinone bypasses the lungs completely. ${ }^{8}$

Inhaled nitric oxide (iNO) has been used for more than 20 years as a pulmonary vasodilator in both cardiac ${ }^{6}$ and non-cardiac ${ }^{9}$ settings. It has the advantage of being delivered directly to the alveoli without reliance on regional PBF. Because it is administered as a gas, it preferentially vasodilates lung regions that are well aerated, which improves ventilation/perfusion matching and leads to improved oxygenation. ${ }^{10}$ After leaving the lungs, iNO is inactivated by reacting with hemoglobin, resulting in minimal systemic vasodilation. ${ }^{6}$ Avoiding rebound PHTN while withdrawing from iNO remains a concern. ${ }^{8}$

Mercier in 1998 demonstrated that although iNO improved the oxygenation index (OI) in neonates with non-cardiac hypoxic respiratory failure, the efficacy varied with the underlying pathology. For example, persistent pulmonary hypertension of the newborn responded very well to iNO, but those infants with congenital diaphragmatic hernia saw much less benefit. ${ }^{11}$ Survival could be predicted by response to iNO after 30 minutes of exposure. ${ }^{11}$ Dowell in 2017 retrospectively studied children (average age 3) with acute respiratory distress syndrome (ARDS) in the absence of cardiac disease who received iNO and found that 
while not all patients responded to iNO with an improved oxygenation index (OI), those that did had few ventilator days (10 days vs 16$)$ but no change in mortality. ${ }^{9}$

Miller in 2000 used iNO prophylactically in cardiac surgical infants who had lesions associated with increased PBF and pre-op PHTN. Treated patients had lower PVR and had significantly fewer PHTN crisis events after surgery (4 vs 7). ${ }^{6}$ They also achieved extubation criteria sooner, but had unchanged ICU LOS. ${ }^{6}$ Cai in 2008 combined iNO with milrinone in post-op fenestrated Fontan patients with elevated PVR and found that combining iNO with milrinone had an additive effect in reducing the transpulmonary gradient. ${ }^{8}$ Villarreal in 2020 published a meta-analysis showing that children who receive iNO after CPB had decreased pulmonary artery pressure and decreased duration of mechanical ventilation as well as ICU-LOS. There was no impact on hospital LOS. ${ }^{7}$

Wong in 2019 retrospectively reviewed 40,000 pediatric cardiac surgical admissions and found that $1.8 \%$ of admissions received iNO despite having no diagnosed PHTN. ${ }^{12}$ These patients had higher mortality and increased hospital LOS. Of the admissions with PHTN, $12 \%$ received iNO, and these had no significant reduction in hospital LOS or mortality. ${ }^{12}$ Gupta in 2019 examined 100,000 post cardiac surgical admissions, and found that of the $15 \%$ who received iNO, only $25 \%$ of these patients had diagnosed PHTN. Centers with lower volume used iNO more frequently, but nonetheless had higher mortality. ${ }^{13}$ Indiscriminate use of iNO is therefore not associated with improved outcomes.

Besides nitric oxide, infants with hypoxic respiratory failure are treated with high frequency oscillatory ventilation (HFOV). This modality improves oxygenation by optimizing alveolar recruitment. ${ }^{14}$ Bojan in 2011 showed that post cardiac surgical infants with hypoxic respiratory failure who were treated with HFOV had shorter duration of ventilation and shorter ICU LOS compared to a matched cohort managed with conventional ventilation only. ${ }^{14}$ Wang and Li in 2013 studied 64 pediatric patients with ARDS following cardiac surgery who were treated with HFOV. They found a significant reduction in oxygenation index (13.4 vs 9.8) with no significant change in mean arterial pressure or central venous pressure after $24 \mathrm{hrs}$ on HFOV. ${ }^{15}$ Unfortunately, this was accompanied by a $34 \%$ incidence of pneumothorax. ${ }^{15}$

Combining HFOV with iNO to treat hypoxic respiratory failure has been studied in non-cardiac surgical infants. The purported benefit lies from recruitment of alveoli by the HFOV, resulting in better delivery of iNO to the pulmonary vasculature. ${ }^{16}$ Dobyns in 2002 studied 108 patients with an oxygenation index (OI) $>15$ receiving either HFOV or conventional ventilation (unblinded). They received either iNO or placebo gas. She found that the $\mathrm{PaO} 2 / \mathrm{FiO} 2$ ratio (P/F ratio) and OI improved with both iNO and HFOV given separately but saw the most improvement when combined. ${ }^{16}$ Kang in 2013 examined outcomes in non-cardiac surgical neonates with an oxygenation index $>25$ who received either HFOV alone or HFOV + iNO. The neonates receiving combination therapy had shorter ventilation time and less early mortality ( $8 \%$ vs $23 \%) .{ }^{10 .}$

This combination of therapies has not been well studied in post- cardiac pediatric surgical patients until now.

Huang et al (2021) in this issue present a retrospective study of post-cardiac surgical pediatric patients with hypoxemic respiratory failure $(\mathrm{P} / \mathrm{F}$ ratio $<300$ or $\mathrm{OI}>8)$ at a single hospital who had failed conventional ventilation. ${ }^{5}$ All the children had PHTN confirmed by echocardiography pre and post CPB. They had simple left to right shunts (atrial septal defect, VSD, or patent ductus arteriosus) with good surgical repair and acceptable hemodynamic parameters. All the children received intravenous milrinone. 39 patients were treated with HFOV alone and 24 received combined HFOV and iNO. When given, the iNO, was discontinued after 2-3 days. They found that the OI improved in both groups but combining iNO with HFOV resulted in lower OI at 48 hours (7.1 vs 6.1). The $\mathrm{P} / \mathrm{F}$ ratio was also higher in the combination group (243 vs 215). Besides oxygenation indices, the experimental group had a shorter duration of mechanical ventilation (5.6 vs 7 days) and shorter ICU-LOS (9.9 vs 12.8 days). The hospital LOS was not affected, and the complication rate was not significantly different between the groups. ${ }^{5}$

Limitations to the general applicability of this study are the fact that it is retrospective, excludes single ventricle physiology, and includes a large fraction $(45 \%)$ of patients who did not undergo CPB. The use 
of iNO was subject to physician discretion, which introduces the potential for selection bias. The effect of treatment on the right ventricle was not specifically reported. This is unfortunate because the impact of combined therapy on right ventricular function may have had a greater impact on ICU LOS and duration of ventilation than the improvement in oxygenation indices.

Nonetheless, this study advances our knowledge of effective management of hypoxic respiratory failure after pediatric cardiac surgery. Further work in this field should include a prospective randomized trial to ascertain if the reduction in duration in ventilation and ICU LOS persists when use of iNO is randomized and blinded. One would also want to study the optimal duration of iNO therapy. This study discontinued the iNO after 3 days, yet the patients were on average extubated after 5 to 7 days. Use of iNO is complicated by the fact that it must be weaned slowly to prevent rebound PHTN, making the timing of discontinuation important. ${ }^{6}$ The benefit of combined iNO + HFOV to post-cardiac surgical patients with single ventricle physiology without PHTN also remains to be examined.

\section{References}

1. Szekely A, Sapi E, Kiraly L, Szatmari A. Intraoperative and postoperative risk factors for prolonged mechanical ventilation after pediatric cardiac surgery. Pediatric Anesthesia 2006 16: 1166-1175

2. Tabib A, Abrishami SE, Mahdavi M, Mortezaeian H, Totonchi Z. Predictors of prolonged mechanical ventilation in pediatric patients after cardiac surgery for congenital heart disease. Cardiovasc Med 2016 5(3): e30391

3. Pagowska-Klimek I, Pychynska-Pokorska M, Krajewski W, Moll JJ. European J of Cardio-thoracic Surgery 2011 40: 179-184

4. Pasquali SK, He X, Jacobs ML, Shah SS, Peterson ED, Gaies MG, Hall M, Gaynor JW, Hill KD, Mayer JE, Li JS, Jacobs JP. Excess costs associated with complications and prolonged length of stay following congenital heart surgery. Ann Thorac Surg 2014 98(5): 1660-1666

5. Huang ST, Lei YQ, Xie WP, Zheng YR, Chen Q, Cao H. Effect of postoperative administration of inhaled nitric oxide combined with high-frequency oscillatory ventilation in infants with acute hypoxemic respiratory failure and pulmonary hypertension after congenital heart surgery: a retrospective cohort study. J of Cardiac Surgery 2021. In press

6. Miller OI, Tang SF, Keech A, Pigott NB, Beller E, Celermajer DS. Inhaled nitric oxide and prevention of pulmonary hypertension after congenital heart surgery: a randomized double-blind study. Lancet 2000 356: 1464-1469

7. Villarreal EG, Aiello S, Evey LW, Flores S, Loomba RS. Effects of inhaled nitric oxide on haemodynamics and gas exchange in children after having undergone cardiac surgery utilizing cardiopulmonary bypass. Cardiology in the Young 2020 30: 1151-1156

8. Cai J, Su Z, Shi Z, Zhou Y, Xu Z, Xu Z, Yang Y. Nitric oxide and milrinone: combined effect on pulmonary circulation after Fontan-type procedure: a prospective randomized study. Ann Thorac Surg 2008 86: 882-888

9. Dowell JC, Thomas NJ, Yehya N. Association of response to inhaled nitric oxide and duration of mechanical ventilation in pediatric acute respiratory distress syndrome. Pediatr Crit Care Med 2017 18(11): 1019-1026

10. Kang W, Sun H, Chen Y, Xu B, Liu D, Jin J, Guo J, Xiong H. Effect of nitric oxide inhalation combined with high-frequency oscillatory ventilation on the prognosis of neonatal severe hypoxemia. Clin Exp Obstet Gynecol 2015 42(1): 22-25

11. Mercier JC, Lacaze T, Storme L, Roze JC, Dinh-Xuan AT, Dehan M. Disease-related response to inhaled nitric oxide in newborns with severe hypoxaemic respiratory failure. Eur J Pediatr 1998 157: $747-752$

12. Wong J, Loomba RS, Evey L, Bronicki RA, Flores S. Postoperative inhaled nitric oxide does not decrease length of stay in pediatric cardiac surgery admissions. Pediatric Cardiology 2019 40: 15591568

13. Gupta P, Rettiganti M, Wilcox A, Eble BK, Schmitz ML, Zakaria D. Spectrum of off-label nitric oxide utilization after pediatric cardiac surgery among centers of varying surgical volume. J of Intensive 
Care Medicine 2019 34(11) 917-923

14. Bojan M, Gioanni S, Mauriat P, Pouard P. High-frequency oscillatory ventilation and short-term outcome in neonates and infants undergoing cardiac surgery: a propensity score analysis. Critical Care 2011 15(5): R259

15. Li S, Wang X, Li S, Yan J. High-frequency oscillatory ventilation for cardiac surgery children with severe acute respiratory distress syndrome. Pediatric Cardiology 2013 34: 1382-1388

16. Dobyns EL, Anas NG, Fortenberry JD, Deshpande J, Cornfield DN, Tasker RC, Liu P, Eells PL, Griebel J, Kinsella JP, Abman SH. Interactive effects of high-frequency oscillatory ventilation and inhaled nitric oxide in acute hypoxemic respiratory failure in pediatrics. Critical Care Med 2002 30(11) 2425-2429 